\title{
Aplicações da quitosana na pós-colheita de frutas
}

\author{
Applications of chitosan in post-harvest fruit \\ Aplicaciones del quitosano en fruta poscosecha
}

Recebido: 14/01/2022 | Revisado: 22/01/2022 | Aceito: 27/01/2022 | Publicado: 29/01/2022

\author{
Ana Raquel Lopes Soares de Almeida \\ ORCID: https://orcid.org/0000-0002-9151-6736 \\ Universidade Estadual do Ceará, Brasil \\ E-mail: raquel.lopes@aluno.uece.br \\ Oriel Herrera Bonilla \\ ORCID: https://orcid.org/0000-0002-9140-6086 \\ Universidade Estadual do Ceará, Brasil \\ E-mail: oriel.bonilla@uece.br \\ Eliseu Marlônio Pereira de Lucena \\ ORCID: https://orcid.org/0000-0002-8190-1702 \\ Universidade Estadual do Ceará, Brasil \\ E-mail: eliseu.lucena@uece.br
}

\begin{abstract}
Resumo
Visando investigar soluções que melhorem os problemas do processamento e armazenamento de frutas após a colheita o presente trabalho realizou uma revisão bibliográfica acerca do uso da quitosana na qualidade pós-colheita de frutas, apontando os seus principais resultados em diversas pesquisas. Para isto, foram utilizados critérios de inclusão na busca nos bancos de dados bases de dados Portal de Periódicos da Capes, Scielo e Google Acadêmico. O biopolímero é extraído da quitina, encontrado principalmente nas cascas de crustáceos e após as etapas de desproteinização, desmineralização, descoloração e desacetilação e apresenta propriedades de biocompatibilidade, atoxicidade, versatilidade, adsorção, biodegradável, entre outras. Devido a estas é aplicada em diversos setores inclusive na agricultura, atuando na produção vegetal, como bioestimulante, inibindo crescimento de microorganismos e melhorando o crescimento, desenvolvimento e produtividade de diversas plantas. Também, pode ser aplicada em frutas, em função da rápida deterioração que ocorre como consequência das reações durante seu amadurecimento, perdendo a sua qualidade como consequência. Desta forma, foram levantados 11 trabalhos que demonstram que a quitosana possibilita a preservação de diferentes frutas por meio da formação de coberturas ao seu redor, mantendo suas características físicas e físico-químicas, reduzindo infecções, estendendo desta forma a vida útil e o seu período de amadurecimento. Diante disto, possui um desempenho positivo no campo agronômico, em pesquisas com ênfase na pós-colheita, sendo uma notável alternativa as atuais técnicas de uso de agroquímicos.
\end{abstract}

Palavras-chave: Fruto; Biopolímero; Quitina.

\begin{abstract}
Aiming to investigate solutions that improve the problems of processing and storing fruits after harvest, the present work carried out a bibliographical review about the use of chitosan in the postharvest quality of fruits, pointing out its main results in several researches. For this, inclusion criteria were used in the search in the databases of Portal de Periódicos from Capes, Scielo and Google Academic. The biopolymer is extracted from chitin, found mainly in crustacean shells and after the steps of deproteinization, demineralization, discoloration and deacetylation, and presents properties of biocompatibility, nontoxicity, versatility, adsorption, biodegradable, among others. Due to these, it is applied in several sectors, including agriculture, acting in plant production, as a biostimulant, inhibiting the growth of microorganisms and improving the growth, development and productivity of several plants. Also, it can be applied to fruits, due to the rapid deterioration that occurs as a result of reactions during its ripening, losing its quality as a result. Thus, 11 studies were surveyed that demonstrate that chitosan allows the preservation of different fruits through the formation of coverings around it, maintaining its physical and physicochemical characteristics, reducing infections, thus extending the shelf life and its period of ripening. In view of this, it has a positive performance in the agronomic field, in research with an emphasis on post-harvest, being a notable alternative to current techniques for the use of agrochemicals.
\end{abstract}

Keywords: Fruit; Biopolymer; Chitin.

\section{Resumen}

Con el objetivo de investigar soluciones que mejoren los problemas de procesamiento y almacenamiento de frutos después de la cosecha, el presente trabajo realizó una revisión bibliográfica sobre el uso del quitosano en la calidad poscosecha de frutos, señalando sus principales resultados en varias investigaciones. Para ello, se utilizaron criterios 
de inclusión en la búsqueda en las bases de datos del Portal de Periódicos de Capes, Scielo y Google Academic. El biopolímero se extrae de la quitina, que se encuentra principalmente en conchas de crustáceos y luego de los pasos de desproteinización, desmineralización, decoloración y desacetilación, y presenta propiedades de biocompatibilidad, no toxicidad, versatilidad, adsorción, biodegradable, entre otras. Por ello, se aplica en varios sectores, entre ellos el agrícola, actuando en la producción vegetal, como bioestimulante, inhibiendo el crecimiento de microorganismos y mejorando el crecimiento, desarrollo y productividad de varias plantas. Asimismo, se puede aplicar a frutos, debido al rápido deterioro que se produce como consecuencia de reacciones durante su maduración, perdiendo como consecuencia su calidad. Así, se relevaron 11 estudios que demuestran que el quitosano permite la conservación de diferentes frutos mediante la formación de coberturas a su alrededor, manteniendo sus características físicas y fisicoquímicas, reduciendo las infecciones, alargando así la vida útil y su período de maduración. En vista de esto, tiene un desempeño positivo en el campo agronómico, en investigaciones con énfasis en poscosecha, siendo una alternativa notable a las técnicas actuales para el uso de agroquímicos.

Palabras clave: Fruta; Biopolímero; Quitina.

\section{Introdução}

Evidências demonstram que as frutas são componentes integrais da alimentação humana (Da Silva \& Spinelli, 2016). Contudo, durante o processo de amadurecimento ocorrem diversos processos fisiológicos e bioquímicos, com objetivo de torna-las atraentes para facilitar a dispersão de suas sementes, e, devido a isto estão sujeitas a deterioração como consequência da transição, dificultando a conservação destes alimentos, que muitas vezes já chegam aos consumidores em estágio de senescência, além dos danos que também, geralmente, são sofridos durante o manejo após a colheita, reduzindo ainda mais a sua vida útil (Giovannoni et al., 2017).

No processamento após a colheita podem ser utilizados produtos químicos aplicados aos alimentos a fim de estender a vida útil, contudo, muitas vezes são tóxicos, com elevada resistência a degradação e nocivos ao ser humano e ambiente. Diante disto, esforços estão em andamento para desenvolver tecnologias para superar as limitações encontradas no armazenamento e durante o trânsito, que sejam seguras, sustentáveis e acessíveis (Gol \& Rao, 2013). A utilização de biopolímeros pode ser uma alternativa com grande potencial de expansão (Brito et al., 2011), devido a serem biodegradáveis e poderem ser obtidos de fontes naturais, a partir de plantas, animais, algas marinhas e bactérias (Farias et al., 2016, Mariano-Torres et al., 2015), possuindo diversas aplicações, inclusive como recobrimentos de alimentos para agricultura.

A quitosana é um biopolímeros natural que tem atraído grande interesse nas últimas duas décadas. É um material biocompatível, quimicamente versátil e biodegradável, podendo ser encontrada em diversas fontes, diferindo quanto ao tamanho e grau de desacetilação, possuindo aplicações em muitas áreas da indústria farmacêutica, médica, engenharia e alimentos (Kean \& Thanou, 2010, Xia et al., 2011). Na agronomia comprova-se como controle eficiente de microrganismos fitopatógenos durante a pré e pós-colheita, tornando as plantas mais resistentes, também na liberação controlada de fertilizantes agroquímicos, no favorecimento da produção vegetal, na fixação biológica de nitrogênio e atribuindo uma vida útil mais longa aos alimentos revestidos (Berger et al., 2011).

Revestimentos comestíveis podem ser potencialmente utilizados como uma técnica de preservação eletiva para prolongar a vida útil das frutas de interesse comercial, melhorando suas propriedades físicas, químicas e sensórias, prolongando efetivamente os seus atributos de qualidade (Gol \& Rao, 2013).

Neste sentido, o presente estudo objetivou elaborar uma revisão bibliográfica acerca do uso da quitosana na qualidade pós-colheita de frutas, apontando os principais resultados de pesquisas com este biopolímero.

\section{Metodologia}

A pesquisa trata-se de uma revisão de literatura sistemática, baseada na produção do conhecimento sobre as aplicações de quitosana na pós-colheita de frutas. Segundo Gerhardt e Silveira (2009), enquadra-se como uma pesquisa qualitativa, tratando-se dos "aspectos da realidade que não podem ser quantificados, centrando-se na compreensão e explicação 
da dinâmica das relações sociais" (Gerhardt \& Silveira, 2009, p. 32). Seu procedimento é do tipo pesquisa bibliográfica, em que são utilizados materiais já publicados para compor a fundamentação teórica, por meio da avaliação de livros, periódicos, documentos, textos e material disponibilizado online (Fontelles et al., 2009). O tipo de pesquisa quanto à finalidade é básica, objetivando gerar conhecimentos novos sem prever aplicações práticas, quanto a sua natureza é do tipo observacional, em que o investigador não realiza intervenção, ou seja, ele é apenas um expectador dos fatos. O objetivo da pesquisa, se enquadra na pesquisa exploratória, que "tem como objetivo proporcionar maior familiaridade com o problema, com vistas a torná-lo mais explícito" (Gerhardt \& Silveira, 2009, p.35).

Inicialmente, foram definidos os critérios de inclusão para a seleção dos documentos, selecionando estudos publicados entre 2011 e 2021, em inglês, português ou espanhol, disponibilizados nas bases de dados Portal de Periódicos da Capes, Scielo e Google Acadêmico. Os termos de busca foram: Qualidade Pós-colheita; Quitosana; Frutas; Post-harvest Quality; Chitosan; Fruits; Calidad Postcosecha. Como critérios de exclusão foram definidos artigos duplicados (repetições) e artigos que antecederam o tempo estipulado para inclusão ou o idioma estabelecido para busca. Os arquivos foram selecionados, e em seguida realizada a análise e leitura completa daqueles considerados pertinentes ao objetivo deste estudo (Figura 1). Posteriormente os conteúdos foram avaliados e organizados em subtópicos de acordo com as discussões apresentados nos resultados da pesquisa, e foram articulados entre si.

Figura 1: Etapas do levantamento bibliográfico.
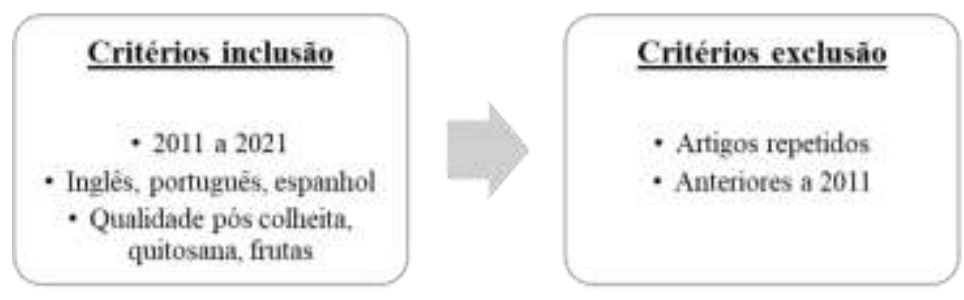

Fonte: Autores.

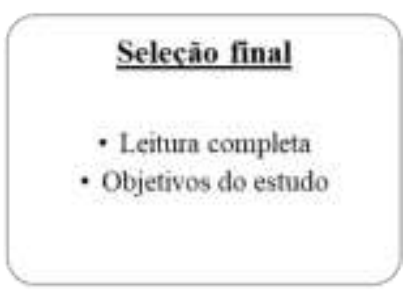

\section{Resultados e Discussão}

\subsection{Qualidade pós-colheita de frutas}

A classificação dos frutos quanto a suas características é complexa. Do ponto de vista botânico, concernem em ovários (órgão feminino da flor) maduros com um tecido acessório não carpelar, os quais se unem após a realização da fecundação e desenvolvimento, abrigando em seu interior a semente (óvulo fecundado), com função de reprodução da planta. De outro modo, genericamente, o termo frutas se refere a frutos consumidos pelo homem, seja resultado da fecundação do ovário, ou de outra parte da flor, ingeridos in natura ou por meio de preparo culinário (Lorenzi et al., 2015). A ambos os termos é designado a função de proteção de sementes e a realização da dispersão destes nos mais variados ambientes (Souza et al., 2019).

As frutas são mundialmente consumidas devido as suas propriedades e valor nutricional (Slavin \& Lloyd, 2012). No entanto, ao ser realizada a colheita, ocorre a interrupção de seu equilíbrio fisiológico original e as células internas passam a não serem mais renovadas, ocorrendo mudanças em suas atividades respiratórias e nos processos metabólicos que são acelerados (Assis \& Brito, 2014). Devido a isto, são bastante perecíveis, continuando progressivamente seus processos de maturação, amadurecimento e senescência, perdendo por sua vez a qualidade com o passar do tempo (Neves, 2016).

A fruticultura é uma das áreas mais importantes na agricultura e a conservação das frutas se destaca como um dos seus maiores desafios, principalmente devido aos diversos problemas fitossanitários, decorrentes do ataque de pragas e doenças (Leite et al., 2016), também ocasionados devido ao desconhecimento de sua fisiologia e a deficiência de tecnologias para o seu 
aproveitamento adequado, que têm contribuído para os altos índices de percas após a colheita (Barbosa et al., 2014). Geralmente ocorrem principalmente devido a colheita realizada fora de época, danos mecânicos no processamento, embalagens inapropriadas; manuseio e transporte inadequados; tempo de exposição prolongado em nível de varejo (Alves et al., 2002).

Desta forma, o interesse por desenvolvimento neste setor vem aumentando, devido ao crescimento das safras e da necessidade de um abastecimento constante de frutas frescas e em boas condições para consumo no mercado interno ou exportação (Neves, 2016). Um dos métodos mais importantes para a conservação de frutas que possibilita a comercialização por diferentes vias de transporte é o armazenamento refrigerado. Este possibilita minimizar as alterações metabólicas e fisiológicas pela diminuição da temperatura, reduzindo rapidamente a respiração dos frutos, retardando o amadurecimento, o murchamento, e a degradação pelo crescimento de microorganismos, consequentemente a sua deterioração (Chitarra \& Chitarra, 2005, Carnelossi et al., 2004, Pinto et al., 2012).

Também com objetivo de conservar estes alimentos devem ser utilizados métodos complementares a refrigeração, em geral, é realizado a aplicação de ceras ou fungicidas, porém o uso de controles químicos está sendo progressivamente proibido em diversos países por serem potencialmente prejudiciais ao homem, causando contaminação no ambiente, afetando o seu processo de amadurecimento, podendo até mesmo ocasionar o surgimento de espécies resistentes de microorganismos (Sasaki et al., 2018 \& Pignati et al., 2017). Produtos de origem natural, provenientes de biopolímeros, podem ser usados como alternativas aos produtos químicos, devido a serem encontrados na natureza, possuírem ampla disponibilidade na natureza e serem biodegradáveis (Luvielmo \& Lamas, 2012).

\subsection{Caracterização da quitosana}

A quitosana (Figura 2B) é um aminopolissacarídeo de cadeias lineares que contêm proporções variáveis dos carboidratos 2-acetamido-2-deoxi-D-glicopiranose e 2-amino-2-deoxi-D-glicopiranose, unidos por ligações glicosídicas (Signini, Campana Filho, 1998). É obtida da quitina (Figura 2A), a partir do exoesqueleto de artrópodes provenientes da indústria pesqueira, animais marinhos ou da parede celular de fungos (Frágas et al., 2015). É o segundo biopolímero mais abundantes na natureza e possui grande potencial para utilização em diversos setores devido as suas características (Silva et al., 2015).

Figura 2: Estrutura química da quitina (A) ( $\beta$-D-glicosamina) e quitosana (B) (N-acetil-D-glicosamina).

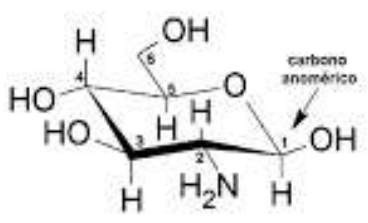

(A) B-D-glicosamina (GlcN)

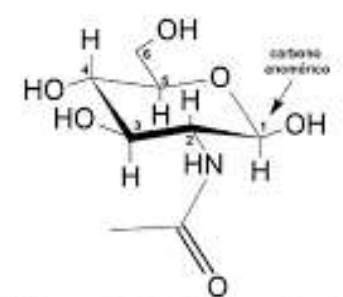

(B) N-acetil-D-glicosamina (GlcNac)

Fonte: Felipe et al. (2017).

Ainda que a quitina seja um polímero natural, o processo de biodegradação das cascas de crustáceos é bastante demorado, gerando grandes quantidades de resíduos de processamento, em média, a indústria de frutos do mar produz 80.000 toneladas de lixo por ano, em média $40 \%$ do seu peso são sobras não utilizadas. O descarte destes produtos é um grande problema mundial enfrentado pela indústria pesqueira e tem levado a esforços de conversão de resíduos em produtos de valor agregado, como a quitosana e seus derivados (Divya \& Jisha, 2017). 
Para a utilização da quitosana é necessário que seja extraída da quitina por métodos químicos ou biológicos, no entanto, as técnicas encontradas na literatura são muito variadas, pois dependem das características da fonte e de sua composição, devido à matéria prima variar muito de uma espécie para outra. Contudo, para o processo químico o resíduo passará por etapas de desproteinização, desmineralização, descoloração e desacetilação da quitina (Kuniyoshi, 2012).

A qualidade final dependerá das condições do processo, assim como das características da fonte, obtendo-se a quitosana com diferentes graus de desacetilação, em geral entre 70 e 90\%, solubilização e de peso molecular (Dias et al., 2013; Tanganini, 2019). A viscosidade, força iônica, pH, e temperatura também dependeram das condições em que ocorreu o processo de extração. De modo geral esta é solúvel em meio aquoso ácido, porém insolúvel em meios neutros e alcalinos (Signini et al., 1998; Abreu et al., 2013, Brugnerotto et al., 2001, Hossain \& Iqbal, 2016, Rabea et al., 2003 \& Rolim et al., 2018).

A quitosana é um polímero biocompatível, não tóxico, quimicamente versátil, e por ser um polieletrólito catiônico possui capacidade de adsorção e habilidade quelante. Além de ser coagulante, acelerador da cicatrização, reabsorvível, biodegradável, solúvel, facilmente processável, fácil de manusear e não alergênico com atividade antimicrobiana (Kean \& Thanou, 2010). Conforme Kuniyoshi (2012), Felipe, Rabelo e Santos (2017), essas características, e a capacidade de interagir com diferentes substancias facilitam seu processamento e o tornam um biomaterial com inúmeras aplicações (Tabela 1).

Tabela 1: Aplicações da quitina e quitosana.

\begin{tabular}{c}
\hline Aplicações da quitosana em diversos setores \\
\hline Implantes cirúrgicos; \\
Recobrimentos de feridas; \\
Liberação controlada de remédios; \\
Cremes medicinais; \\
Suplementos para dietas; \\
Cosméticos; \\
Recobrimentos de alimentos; \\
Tratamento de água; \\
Inseticidas; \\
Agricultura.
\end{tabular}

Fonte: Adaptada de Kuniyoshi (2012).

Devido ao baixo custo deste polímero existe cada vez mais interesse de sua atuação na área médica e odontológica, para tratamento de lesões cutâneas, em suturas cirúrgicas, implantes dentários, reconstituição óssea, lentes de contato, também na farmacêutica, por meio da liberação controlada de drogas, encapsulamento de materiais, devido suas propriedades anticoagulantes, antitumoral, imunológico e hemostático. Também na indústria de alimentos, atuando como redutor de colesterol, conservante, fungicida e bactericida e recobrimento de frutas, na indústria de cosméticos, como esfoliantes, hidratantes, cremes dentais e tratamento de acne. Outra área em que possui diversas aplicações é no tratamento de água, através da descontaminação de efluentes industriais (Azevedo et al., 2007, Berger et al., 2011, Signini et al., 1998 \& Frágas et al., 2015).

$\mathrm{Na}$ agricultura é utilizada principalmente, para aumentar o rendimento das culturas, no controle de doenças de plantas e resistência ao estresse, devido a capacidade antimicrobiana e como um regulador de genes responsáveis pela produção de enzimas de resistência a induzir imunidade nas plantas, desta forma, inibindo viroses e outras infecções bacterianas ou fúngicas. Pode ser utilizada ainda no revestimento de sementes, para o enriquecimento do solo, na liberação lenta de biopesticidas e na pulverização foliar (Morin-Crini et al., 2019). Além disto, devido ao seu caráter hidrofílico, formam 
coberturas comestíveis, ajudando na preservação de frutas, prevenindo infecções e melhorando a qualidade de alimentos, cujo tempo de prateleira é consideravelmente reduzido (Assis, Brito, 2014, Kuniyoshi, 2012 \& Sharif et al., 2018).

\subsection{Aplicação de quitosana na qualidade pós-colheita de frutas}

Entre os polissacarídeos naturais a quitosana se destaca, por ser amplamente utilizada na conservação de alimentos (Sena et al., 2016). Sua estrutura polimérica gelificada possibilita sua aplicação sob a forma de filmes comestíveis, películas sólidas com espessura variada que podem, geralmente, ser consumidas (Maia et al., 2000), ou revestimentos comestíveis, que são formados a partir de soluções aplicadas a superfície das frutas, ficando em contato íntimo com estas (Dhall, 2013).

A aplicação destas coberturas requer conhecimentos da formulação da solução e de seu modo de degradação, assim como dos aspectos fisiológicos e metabólicos da fruta estudada (Chitarra \& Chitarra, 2005), e devido as diferenças fisiológicas presentes nelas a aplicação de diferentes tipos de coberturas, assim como de concentrações, é muito pesquisada, com objetivo de desenvolver técnicas mais benéficas ao aumento da vida útil (Luvielmo \& Lamas, 2012).

Desta forma foram analisadas 11 pesquisas (Quadro 1) sobre o uso da quitosana na qualidade pós-colheita de frutas, levantando diversas espécies de interesse alimentício que são exóticas e nativas do Brasil, observando os principais resultados, solução com melhor desempenho e vida útil máxima com a solução filmogênica. O polissacarídeo demostrou resultados satisfatórios na qualidade pós-colheita do mamão (Galo et al., 2014), da banana (Hossain \& Iqbal, 2016 \& Jafarizadeh, 2011), no caju (Ferreira, Santos, Moreira, Atílio \& Conceição, 2010), na mangaba (Nasser et al., 2016), carambola (Sanches et al., 2017), goiaba (Soares et al., 2011), maçã (Castañeda, 2013, Jorge et al., 2011), morango (Melo, 2020) e na manga (Souza et al., 2011). 
Quadro 1: Estudos feitos com quitosana como coberturas de frutos.

\begin{tabular}{|c|c|c|c|c|}
\hline Frutas em estudo & $\begin{array}{l}\text { Solução com melhor } \\
\text { desempenho }\end{array}$ & Principais resultados & $\begin{array}{l}\text { Vida útil } \\
\text { máxima com } \\
\text { a solução de } \\
\text { quitosana }\end{array}$ & Autores \\
\hline $\begin{array}{l}\text { Carica papaya } \mathrm{L} \text {. } \\
\text { (Mamão 'Sunrise } \\
\text { solo') }\end{array}$ & $1 \%$ & $\begin{array}{l}\text { Aumento proporcional dos teores de acidez titulável } \\
\text { (ATT), sólidos solúveis totais (SST) e vitamina C. }\end{array}$ & 13 dias & $\begin{array}{l}\text { Galo et al. } \\
\text { (2014) }\end{array}$ \\
\hline $\begin{array}{c}\text { Musa acuminata } \\
\text { Colla (Grupo } \\
\text { Cavendish) (Banana } \\
\text { 'Beragan') } \\
\end{array}$ & $2,02 \%$ & $\begin{array}{c}\text { Houve uma menor perca de peso, da firmeza, e } \\
\text { mudança de coloração na casca dos frutos tratados, } \\
\text { assim como estabilização das concentrações de acidez } \\
\text { titulável total. }\end{array}$ & 10 dias & $\begin{array}{l}\text { Jafarizadeh } \\
\text { et al., } 2011\end{array}$ \\
\hline $\begin{array}{l}\text { Musa sapientum } \mathrm{L} . \\
\text { (Banana } \\
\text { 'Amritasagar') }\end{array}$ & $1 \%$ & $\begin{array}{c}\text { Menor perca de peso, da cor da casca, estabilização das } \\
\text { concentrações de acidez titulável total e sólidos } \\
\text { solúveis totais. }\end{array}$ & 16 dias & $\begin{array}{l}\text { Hossain \& } \\
\text { Iqbal, } 2016\end{array}$ \\
\hline $\begin{array}{l}\text { Anacardium } \\
\text { occidentale } \mathrm{L} . \\
\text { (Caju) }\end{array}$ & $5 \%$ & $\begin{array}{c}\text { Não demonstrou resultados significativos na perca de } \\
\text { massa, sólidos solúveis totais, pH, da ácidez titulável } \\
\text { total e vitamina C. }\end{array}$ & 6 dias & $\begin{array}{l}\text { Ferreira et } \\
\text { al., } 2010\end{array}$ \\
\hline $\begin{array}{l}\text { Hancornia specios } \\
\text { Gomes (Mangaba) }\end{array}$ & $2 \%$ & $\begin{array}{l}\text { Os frutos tratados tiveram menor perca de peso, } \\
\text { menores danos visuais ao fim armazenamento e } \\
\text { diminuição dos teores de açúcar redutor. }\end{array}$ & 15 dias & $\begin{array}{l}\text { Nasser et } \\
\text { al., } 2016\end{array}$ \\
\hline $\begin{array}{l}\text { Averrhoa carambola } \\
\text { L. (Carambola) }\end{array}$ & $2 \%$ e $3 \%$ & $\begin{array}{l}\text { Menor perca de peso, estabilização dos teores de } \\
\text { sólidos solúveis totais e pH, regressão da firmeza, } \\
\text { vitamina C e da concentração de acidez titulável total. }\end{array}$ & 12 dias & $\begin{array}{l}\text { Sanches et } \\
\text { al., } 2017\end{array}$ \\
\hline $\begin{array}{l}\text { Pisidium guajava L. } \\
\text { (Goiaba 'Pedro } \\
\text { Sato') }\end{array}$ & $1 \%$ e $1,5 \%$ & $\begin{array}{l}\text { Menor mudança da coloração casca e polpa, e do teor } \\
\text { de sólidos solúveis totais. }\end{array}$ & 12 dias & $\begin{array}{l}\text { Soares et al., } \\
\quad 2011\end{array}$ \\
\hline $\begin{array}{l}\text { Malus domestica } \\
\text { Borkh (Maça 'Royal } \\
\text { Gala') }\end{array}$ & $0,2 \%$ & $\begin{array}{l}\text { Retardo da maturação, da perca de firmeza e de } \\
\text { vitamina C. Possuíram luminosidade elevada, melhora } \\
\text { da aparência e textura dos frutos. }\end{array}$ & 28 dias & $\begin{array}{l}\text { Jorge et al., } \\
\quad 2011\end{array}$ \\
\hline $\begin{array}{l}\text { Mangifera indica L. } \\
\text { (Manga 'Tommy } \\
\text { Atkins') } \\
\end{array}$ & $1,5 \%$ & $\begin{array}{l}\text { O revestimento retardou sd mudanças de cor da polpa, } \\
\text { dos teores de sólidos solúveis totais, acidez titulável } \\
\text { total relação SST/ATT, vitamina C e da firmeza. }\end{array}$ & 9 dias & $\begin{array}{l}\text { Souza et al., } \\
\quad 2011\end{array}$ \\
\hline $\begin{array}{l}\text { Malus domestica } \\
\text { Borkh (Maça 'Fuji') }\end{array}$ & $1 \%$ e $2 \%$ & $\begin{array}{l}\text { Redução da perca de peso e da firmeza melhora da } \\
\text { aparência, promovendo mais brilho e constância dos } \\
\text { teores de sólidos solúveis totais e acidez titulável total. }\end{array}$ & 20 dias & $\begin{array}{l}\text { Castañeda, } \\
2013\end{array}$ \\
\hline $\begin{array}{l}\text { Fragaria } x \\
\text { Ananassa Duch. } \\
\quad \text { (Morango) }\end{array}$ & $\begin{array}{l}\text { Gel com nanopartículas } \\
\text { de quitosana com } \\
\text { tamanho }(331,1 \mathrm{~nm}), \\
\text { potencial zeta }(+34 \mathrm{mV}) \\
\text { e PDI }(0,377)\end{array}$ & $\begin{array}{l}\text { A cobertura atuou como barreira à perda de umidade e } \\
\text { de peso mantendo o teor de sólidos solúveis e o índice } \\
\text { de maturação das frutas, preservando a cor, } \\
\text { antocianina, pH e acidez titulável total. Apresentaram } \\
\text { também importante atividade antioxidante e } \\
\text { antimicrobiana, inibindo o crescimento in vivo e in } \\
\text { vitro dos fungos fitopatogênicos. }\end{array}$ & 12 dias & Melo, 2020 \\
\hline
\end{tabular}

Fonte: Autores.

As soluções de quitosana foram aplicadas por imersão na maioria dos frutos, exceto na mangaba (Nasser et al., 2016) em que foi utilizada a técnica de pulverização. Entre as técnicas de aplicação de coberturas, a imersão possibilita melhor que toda a superfície do alimento entre em contato com a solução, possibilitando a deposição uniforme, enquanto que a pulverização pode depender das condições de aplicação (Assis \& Brito, 2014). Após a secagem das coberturas, a maioria dos autores realizou o armazenamento os frutos a temperatura de 20 a $26^{\circ} \mathrm{C}$, contudo, para carambola (Sanches et al., 2017), goiaba 'pedro sato' (Soares et al., 2011), 'maça fuji' (Castãneda, 2013), caju (Ferreira et al., 2010) e mangaba (Nasser et al., 2016) foi realizado armazenamento refrigerado com temperatura específica para cada um.

Através da análise dos resultados obtêm-se que a quitosana atribui uma vida útil mais longa as frutas revestidas, mantendo a integridade mecânica e melhorando as suas características físicas e químicas, retardando a mudança de coloração da casca e polpa, reduzindo a perca de umidade, a migração dos solutos, as trocas gasosas, respiração e velocidade de reação de oxidação, os distúrbios fisiológicos, inibindo o crescimento de microorganismos, mantendo a firmeza e retardando o 
murchamento das frutas, nos processos metabólicos que atrasam o amadurecimento (Chevalier et al., 2016, Sanches et al., 2017).

\section{Conclusão}

Depreende-se, por meio da revisão, que a utilização do polissacarídeo natural quitosana é efetiva na pós-colheita de frutas quando utilizado como uma cobertura para conservar e aumentar a vida útil destes alimentos. Recomenda-se mais estudos sobre o seu uso, buscando compreender de que outras formas atuam em seus diferentes atributos de qualidade, superando as dificuldades fitossanitárias encontradas na fruticultura.

Sugere-se para trabalhos futuros, pesquisar a ação da quitosana associada aos óleos essenciais para a aplicação na póscolheita de frutas, de tal forma que as limitações possam ser suplantadas, melhorando as características do biofilme, bem como, demonstrando a sua utilização por meio de outras técnicas de aplicação que não sejam exclusivamente a imersão, buscando metodologias que sejam sustentáveis, seguras e de baixo custo.

\section{Agradecimentos}

À Fundação Cearense de Apoio ao Desenvolvimento Científico e Tecnológico (FUNCAP), pela concessão da bolsa de mestrado ao primeiro autor.

\section{Referências}

Abreu, F. O., Cavalcante, L. G., Doudement, P. V., Castro, A. M., Nascimento, A. P. D., \& Matos, J. E. (2013). Propriedades e características da quitosana obtida a partir do exoesqueleto de caranguejo-uçá utilizando radiação de microondas. Polímeros, 23(5), 630-635. https://www.scielo.br/scielo.php?pid=S0104$14282013000500011 \&$ script=sci_arttext

Alves, R. E., Filgueiras, H. A. C., Menezes, J. B., de Assis, J. S., de Lima, M. A. C., Amorim, T. B. F., \& Martins, A. G. (2002). Colheita e pós-colheita: a cultura da mangueira. Embrapa Semiárido, ALICE.

Assis, O. B. G., \& Britto, D. D. (2014). Revisão: coberturas comestíveis protetoras em frutas: fundamentos e aplicações. Brazilian Journal of Food Technology, 17(2), 87-97. https://www.scielo.br/scielo.php?pid=S1981-67232014000200001\&script=sci_arttext

Azevedo, V., Chaves, S. A., Bezerra, D. C., Lia Fook, M. V., \& Costa, A. C. F. M. (2007). Quitina e Quitosana: aplicações como biomateriais. Revista eletrônica de Materiais e processos, 2(3), 27-34. https://www2.ibb.unesp.br/Museu_Escola/4_diversidade/alimentacao/Textos/Quitina_Quitosana.pdf

Berger, L. R. R., Stamford, T. C. M., \& Stamford, N. P. (2011). Perspectivas para o uso da quitosana na agricultura. Revista Iberoamericana de Polimeros, 12(4), 195-215. http://www.ehu.eus/reviberpol/pdf/AGO11/ramos.pdf

Brito, G. F., Agrawal, P., Araújo, E. M., \& Mélo, T. J. A. (2011). Biopolímeros, polímeros biodegradáveis e polímeros verdes. Revista eletrônica de materiais e Processos, 6(2), 127-139. http://www2.ufcg.edu.br/revista-remap/index.php/REMAP/article/download/222/204

Brugnerotto, J., Lizardi, J., Goycoolea, F. M., Argüelles-Monal, W., Desbrieres, J., \& Rinaudo, M. (2001). An infrared investigation in relation with chitin and chitosan characterization. Polymer, 42(8), 3569-3580. https://www.sciencedirect.com/science/article/pii/S0032386100007138?casa_token =BJCsE10kSB0AAAAA:w9tDMN07bBQpQakHf0LQbU_oXU1PjKoEfLug3AyGUk32LnOwqJrdwQxhh2anmU48NVm7AgFs8eE

Carnelossi, M. A. G., Toledo, W. F. F., Souza, D. C. L., Lira, M. D. L., Silva, G. F. D., Jalali, V. R. R., \& Viégas, P. R. D. A. (2004). Conservação póscolheita de mangaba (Hancornia speciosa Gomes). Ciência e $\quad$ Agrotecnologia, https://www.scielo.br/j/cagro/a/dsvGBWXXX96LgTsg8hCn3Wf/abstract/?lang=pt

Castañeda, L. M. F. (2013). Avaliação da quitosana e da fécula de mandioca, aplicada em pós-colheita no recobrimento de maçãs (Tese de Doutorado). Universidade do Rio Grande do Sul, RS.

Chevalier, R. C., Alves da Silva, G. F., Silva, D. M. D., Pizato, S., \& Cortez-Vega, W. R. (2016). Edible coating utilization the chitosan base to improve melon shelf life minimally processed. Journal of bioenergy and food science, 130-138. https://repositorio.unesp.br/handle/11449/159266

Chitarra, M. I. F., \& Chitarra, A. B. (2005). Pós-colheita de frutos e hortaliças: fisiologia e manuseio. Esal/Faepe.

de Barbosa, S. L., Macedo, J. L., dos Santos, C. M., \& Machado, A. V. (2014). Estudo da secagem de frutos tropicais do Nordeste. Revista Verde de Agroecologia e Desenvolvimento Sustentável, 9(1), 186-190. https://editoraverde.org/gvaa.com.br/revista/index.php/RVADS/article/view/2702

de Farias, S. S., Siqueira, S. M. C., Cristino, J. H. S., \& da Rocha, J. M. (2016). Biopolímeros: uma alternativa para promoção do desenvolvimento sustentável. Revista Geonorte, 7(26), 61-77. https://periodicos.ufam.edu.br/revista-geonorte/article/view/2759 
da Silva, S. B., \& Spinelli, M. G. N. (2015). Consumo de frutas em unidade de alimentação e nutrição no município de São Paulo: um estudo de caso. Revista Univap, 21(38), 5-14. http://revista.univap.br/index.php/revistaunivap/article/view/292

Dhall, R. K. (2013). Advances in edible coatings for fresh fruits and vegetables: a review. Critical reviews in food science and nutrition, 53(5), 435-450. https://www.tandfonline.com/doi/abs/10.1080/10408398.2010.541568

Dias, K. B., Silva, D. D., Ferreira, L. A., Fidelis, R. R., Costa, J. D. L., Silva, A. D., \& Scheidt, G. N. (2013). Quitina e quitosana: características, utilizações e perspectivas atuais de produção. Journal of Biotechnology, 4(3), 184-191. https://sistemas.uft.edu.br/periodicos/index.php/JBB/article/view/599

Divya, K. \& Jisha, M. S. (2018). Chitosan nanoparticles preparation and applications, Environmental chemistry letters, 16(1), 101-112. https://link.springer.com/article/10.1007/s10311-017-0670-y

Felipe, L. O., Rabello, L. A., Júnior, E. N. O., \& Santos, I. J. B. (2017). Quitosana: da química básica à bioengenharia. Química Nova na escola, 39(4), 312320. https://revistas.ufpr.br/academica/article/view/570

Ferreira, A. P. S., Santos, I. J., Moreira, E. R., Atílio, L. B., \& Conceição, B. A. (2010). Qualidade pós-colheita do pseudofruto do cajueiro sob ação de quitosana e refrigeração. Revista Cultura Agronômica, 19(1), 64-70. https://ojs.unesp.br/index.php/rculturaagronomica/article/view/2168

Fontelles, M. J., Simões, M. G., Farias, S. H., \& Fontelles, R. G. S. (2009). Metodologia da pesquisa científica: diretrizes para a elaboração de um protocolo de pesquisa. Revista paraense de medicina, 23(3), 1-8. https://files.cercomp.ufg.br/weby/up/150/o/Anexo_C8_NONAME.pdf

Fráguas, R. M., Rocha, D. A., Queiroz, E. D. R., Abreu, C. M. P. D., Sousa, R. V. D., \& Oliveira, E. N. D. (2015). Caracterização química e efeito cicatrizante de quitosana, com baixos valores de massa molar e grau de acetilação, em lesões cutâneas. Polímeros, 25, 205-211. https://www.scielo.br/j/po/a/y78Pk5934TvBtjyxNXVPXYH/?lang=pt\&format=html

Galo, J. D. Q. B., Souza, M. L. D., Kusdra, J. F., \& Mattiuz, C. F. M. (2014). Postharvest storage of papaya 'Sunrise Solo' with the use of chitosan. Revista Brasileira de Fruticultura, 36(2), 305-312. https://www.scielo.br/scielo.php?pid=S0100-29452014000200005\&script=sci_arttext\&tlng=pt

Gerhardt, T. E. \& Silveira, D. T. (2009). Métodos de Pesquisa. Editora da UFRGS. p. 120.

Giovannoni, J., Nguyen, C., Ampofo, B., Zhong, S., \& Fei, Z. (2017). The epigenome and transcriptional dynamics of fruit ripening. Annual Review of Plant Biology, 68, 61-84. https://www.annualreviews.org/doi/abs/10.1146/annurev-arplant-042916-040906?casa_token=LYPwCrYErSYAAAAA:Zw OfYmSqLxVUQvAsG3fcto0OEW6CZoMNUj817hDobJz1113O9ycXmSTr9aXUAiVZocUh3ijnmHpLKF6wEA

Gol, N. B., \& Rao, T. R. (2014). Influence of zein and gelatin coatings on the postharvest quality and shelf life extension of mango (Mangifera indica L.). Fruits, 69(2), 101-115. https://www.cambridge.org/core/journals/fruits/article/influence-of-zein-and-gelatin-coatings-on-the-postharvest-quality-and-shelf-lifeextension-of-mango-mangifera-indica-1/FAE8D3801D5CBF0C8B96B7181752ACFC

Hossain, M. S., \& Iqbal, A. (2016). Effect of shrimp chitosan coating on postharvest quality of banana (Musa sapientum L.) fruits. International Food Research Journal, 23(1), 277. http://ifrj.upm.edu.my/23\%20(01)\%202016/(41).pdf

Jafarizadeh, M. H., Osman, A., Tan, C. P., \& Abdul, R. (2011). Development of an edible coating based on chitosan-glycerol to delay Berangan banana (Musa

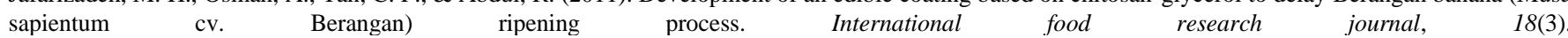
http://search.ebscohost.com/login.aspx?direct=true\&profile=ehost\&scope=site \&authtype=crawler \&jrnl=19854668\&AN=64500102\&h=W0UkjIVGCWvbUF OstM7arak3SguKvV83sIM5AD\%2Fa8b6TfaYGnFxobgWWgNiu506YsWCvxZj9qhKTk3L1XC2RLw\%3D\%3D\&crl=c\&casa_token=cugs06gPfc0AAAAA: w_175kIPJSsKK5YRKB2olHULjo5TnfrtD1jQah38e83oRfINVv69u1DwXphE4kByKwY9qjtDBpuJAZx-

Jorge, P. C. S., Nucci, M., Rizzo, J. S., Assis, O. B. G., \& Monteiro, M. (2011). Maçã "Royal Gala" revestida com quitosana estocada à temperatura ambiente. Boletim do Centro de Pesquisa $e$ Processamento 29 de 253-264. https://www.researchgate.net/profile/Magali_Monteiro/publication/262957390_Shelf_Life_of_Fresh_and_Pasteurized_Organic_Passion_Fruit_Passiflora_Edu lis_F_Flavicarpa_Deg_Pulp/links/56af5a0a08ae28588c62fca7/Shelf-Life-of-Fresh-and-Pasteurized-Organic-Passion-Fruit-Passiflora-Edulis-F-FlavicarpaDeg-Pulp.pdf

Kean, T., \& Thanou, M. (2010). Biodegradation, biodistribution and toxicity of chitosan. Advanced drug delivery reviews, 62(1), 3-11. https://www.sciencedirect.com/science/article/pii/S0169409X0900283X?casa_token=73Eb1Z-u9j0AAAAA:SX_tVOy92k1LoFPURe4X2gyEX2dHexu02qrQ0DRa5NitBrMDtlN5sNZ1fkgpIMswmT7rctIRu0

Kuniyoshi, J. N. La quitosana. (2012) Revista de química PUCP, 26(12), 10-12. http://revistas.pucp.edu.pe/index.php/quimica/article/view/6421

Leite, S. A., Castellani, M. A., Ribeiro, A. E. L., Moreira, A. A., \& Aguiar, W. M. M. (2016). Perfil dos fruticultores e diagnóstico do uso de agrotóxicos no polo de fruticultura de Livramento de Nossa Senhora, Bahia. Extensão Rural, 23(2), 112-125. https://periodicos.ufsm.br/extensaorural/article/view/13538

Lorenzi, H.,Lacerda, M. T. C. \& Bacher, L. B (2015). Frutas no Brasil: nativas e exóticas (de consumo in natura). Instituto Plantarum de Estudos da Flora.

Luvielmo, M. M., \& Vieira Lamas, S. (2012). Revestimentos comestíveis em frutas. Estudos Tecnológicos em Engenharia, 8(1). https://core.ac.uk/download/pdf/228891333.pdf

Maia, L. H., Porte, A., \& Souza, V. F. (2000). Filmes comestíveis: aspectos gerais, propriedades de barreira a umidade e oxigênio. Boletim do Centro de Pesquisa de Processamento de Alimentos, 18(1). https://revistas.ufpr.br/alimentos/article/download/1129/930

Mariano-Torres, J. A., López-Marure, A., \& Domiguez-Sánchez, M. Á.(2015). Síntesis y caracterización de polímeros a base de ácido cítrico y glicerol: Su aplicación en polímeros não biodegradáveis: Su aplicación en polímeros não biodegradáveis. Dyna, 82(190), 53-59. https://ipn.elsevierpure.com/en/publications/synthesis-and-characterization-of-polymers-based-on-citric-acid-a

Melo, N. F. C. B. (2020). Bioatividade de coberturas comestíveis a base de quitosana em gel e nanopartículas na conservação de morangos (Fragaria $x$ ananassa) (Tese de Doutorado), Universidade Federal de Pernanbuco, PE. 
Morin-Crini, N., Lichtfouse, E., Torri, G., \& Crini, G. (2019). Applications of chitosan in food, pharmaceuticals, medicine, cosmetics, agriculture, textiles, pulp and paper, biotechnology, and environmental chemistry. Environmental Chemistry Letters, 17(4), 1667-1692. https://link.springer.com/article/10.1007/s10311-019-00904-x

Nasser, F. A. D. C. M., Boliani, A. C., Nasser, M. D., Pagliarini, M. K., \& Mendonça, V. Z. (2016). Conservação de mangabas submetidas à aplicação de quitosana. Científica, 44(3), 279-285. http://cientifica.org.br/index.php/cientifica/article/view/793

Neves, L. C (2016). Manual pós-colheita da fruticultura brasileira. Eduel.

Pignati, W. A., Lima, F. A. N. D. S., Lara, S. S. D., Correa, M. L. M., Barbosa, J. R., Leão, L. H. D. C., \& Pignatti, M. G. (2017). Distribuição espacial do uso de agrotóxicos no Brasil: uma ferramenta para a Vigilância em Saúde. Ciência \& Saúde Coletiva, 22, 3281-3293. https://www.scielosp.org/article/csc/2017.v22n10/3281-3293/

Pinto, J. A. V., Brackmann, A., Schorr, M. R. W., Venturini, T. L., \& Thewes, F. R. (2012). Indução de perda de massa na qualidade pós-colheita de Pêssegos 'Eragil' em armazenamento refrigerado. Ciência Rural, 42, 962-968. https://www.scielo.br/j/cr/a/4KnjWpKSPgLQtfBSK9nXs6C/?format=html\&lang=pt

Rabea, E. I., Badawy, M. E. T., Stevens, C. V., Smagghe, G., \& Steurbaut, W. (2003). Chitosan as antimicrobial agent: applications and mode of action. Biomacromolecules, 4(6), 1457-1465. https://pubs.acs.org/doi/abs/10.1021/bm034130m?casa_token=fOCx0HxEjuoAAAAA:BjLG9OH-tZJhO8iZzi6i8CjDBVSsCtoDDSeWL4z0zTeqXVIscjYKPK8eNfFd0NvORXAb2YmbVr6Em9U

Rolim, A. E. H., Carvalho, vF. A., Costa, R. C. C., \& Rosa, F. P. (2018). Arcabouços de quitosana-propriedades físico-químicas e biológicas para o reparo ósseo. Rev.Virtual. Quim, 10. https://www.researchgate.net/profile/Ana_Emilia_Rolim/publication/325160842_Chitosan_Scaffolds_-_Physico_-

Sanches, A. G., da Silva, M. B., Moreira, E. G. S., \& Cordeiro, C. A. M. (2018). Preservação da qualidade pós-colheita da carambola com solução filmogênica de quitosana. Colloquium Agrariae, 14(2),122-132. http://revistas.unoeste.br/index.php/ca/article/view/1821

Sasaki, F. F. C., Pereira, M. E. C., de Morais, P. L. D., de Almeida, G. V. B., Terao, D., Oster, A. H., \& Cerqueira, T. S. (2018). Manejo pós-colheita e desenvolvimento de tecnologias para aplicação em pós-colheita para redução do uso de agrotóxicos em mamão. In: Simpósio do papaya brasileiro, Vitória. ES.

Sena, E. D. O. A., Araújo Couto, H. G. S., Paixão, R. C., Silveira, M. P. C., Oliveira Jr., L. F. G., \& Carnelossi, M. A. G. (2016). Utilização de biofilme comestível na conservação pós-colheita de pimentão verde (Capsicum annuum L.). Scientia Plena, 12(8). https://www.scientiaplena.org.br/sp/article/view/2938

Sharif, R., Mujtaba, M., Ur Rahman, M., Shalmani, A., Ahmad, H., Anwar, T. \& Wang, X. (2018). The multifunctional role of chitosan in horticultural crops; a review. Molecules, 23(4), 872. https://www.mdpi.com/1420-3049/23/4/872

Signini, R., \& Campana Filho, S. P. (1998). Purificação e caracterização de quitosana comercial. Polímeros, 8, 63-68. https://www.scielo.br/j/po/a/4s3Nd9ChqH79dqVNT7RyK4d/abstract/?lang=pt

Silva, M. G., Silva, S. B., Silva, J., Santin, C. K., Souza, D., \& Frota Jr, M. (2015). Caracterização de filmes à base de quitosana. In Proceedings of the 9th Congresso Brasileiro de Engenharia Química em Iniciação Científica, São Paulo, SP

Slavin, J. L., \& Lloyd, B. (2012). Health benefits of fruits and vegetables. Advances in nutrition, 3(4), 506-516. https://academic.oup.com/advances/articleabstract/3/4/506/4591497

Soares, N. D. F. F., Silva, D. F. P., Camilloto, G. P., Oliveira, C. P., Pinheiro, N. M., \& Medeiros, E. A. A. (2011). Antimicrobial edible coating in postharvest conservation of guava. Revista Brasileira de Fruticultura, 33(SPE1), 281-289. https://www.scielo.br/scielo.php?pid=S010029452011000500035\&script=sci_arttext\&tlng=pt

Souza, M. L. D., Morgado, C. M. A., Marques, K. M., Mattiuz, C. F. M., \& Mattiuz, B. H. (2011). Pós-colheita de mangas' Tommy Atkins' recobertas com quitosana. Revista Brasileira de Fruticultura, 337-343. https://repositorio.unesp.br/handle/11449/4166

Souza, V. C., Lorenzi, H. \& Flores, T. B (2019). Introdução à botânica: morfologia. Instituto Plantarum de Estudos da Flora.

Tanganini, I. C. (2019). Extração de quitosana a partir do resíduo de camarão e seu efeito sobre as leveduras do bioetanol. (Dissertação de Mestrado). Universidade Federal de São Carlos, Araras.

Xia, W., Liu, P., Zhang, J., \& Chen, J. (2011). Biological activities of chitosan and chitooligosaccharides. Food hydrocolloids, 25(2), 170-179. https://www.sciencedirect.com/science/article/pii/S0268005X10000469?casa_token=UcztHoDDj7IAAAAA:93IgN6Ds1bNfU_wUei8CRAESrv_jrYcIcFl11J0 VYDY8OMa-ohqg_RQVHXHYVhpKFWqUFxgnHnOo 